\title{
Correlative analyses of the expression levels of PIAS3, p-SHP2, SOCS1 and SOCS3 with STAT3 activation in human astrocytomas
}

\author{
LI-HONG LIU, HONG LI, XIAO-XIN CHENG, QING-YOU KONG, \\ XIAO-YAN CHEN, MO-LI WU, YAN LI, JIA LIU and CONG LI \\ Liaoning Laboratory of Cancer Genetics and Epigenetics, Department of Cell Biology, \\ College of Basic Medical Sciences, Dalian Medical University, Dalian, Liaoning 116044, P.R. China
}

Received November 11, 2015; Accepted November 10, 2016

DOI: $10.3892 / \mathrm{mmr} .2016 .6079$

\begin{abstract}
The importance of signal transducer and activator of transcription 3 (STAT3) signaling in the growth and survival of glioblastoma cells has been well documented, while the reasons leading to STAT3 activation remains to be elucidated. Suppressors of cytokine signaling (SOCS) 1 and SOCS3, SH2 domain-containing phosphatase (SHP2) and protein inhibitors of activated STAT3 (PIAS3) are known to inhibit STAT3 signal transduction, while their expression statuses in the four grades of astrocytomas and relevance with STAT3 activation remain to be described. The present study aimed to address these issues by tissue microarray-based immunohistochemical profiling the expression levels of phosphorylated (p)-STAT3, SOCS1, SOCS3, PIAS3 and p-SHP2. The results revealed that p-STAT3 nuclear translocation was rarely observed in non-cancerous brain tissues and its frequencies were increased in a tumor grade-associated manner $(65.2,77.1,81.8$ and $85.7 \%$ for grade I-IV, respectively). PIAS3, p-SHP2, SOCS1 and SOCS3 were expressed in higher levels $(++$ and +++$)$ in 63.6 , $90,87.5$ and $81.8 \%$ of tumor surrounding brain tissues, which reduced to $13.1,47.8,33.3$ and $50 \%$ in grade I, 11.4, 65.7, 58.3 and $77.1 \%$ in grade II, 9.1, 63.6, 38.1 and $31.8 \%$ in grade III and $7.1,66.7,30.8$ and $7.1 \%$ in grade IV astrocytomas. The above results revealed that although the expression levels of SOCS1, SOCS3 and, in particular, p-SHP2, tend to decrease in the four types of astrocytomas, PIAS3 downregulation is more negatively correlated with STAT3 activation in the stepwise
\end{abstract}

Correspondence to: Professor Jia Liu or Professor Cong Li, Liaoning Laboratory of Cancer Genetics and Epigenetics, Department of Cell Biology, College of Basic Medical Sciences, Dalian Medical University, West Section, 9 Lvshun South Road, Dalian, Liaoning 116044, P.R. China

E-mail: jialiudl@aliyun.com

E-mail: goodluck_licong@163.com

Key words: astrocytomas, STAT3 signaling, STAT3 negative regulators, PIAS3, gene expression progress of astrocytomas and would indicate an unfavorable outcome.

\section{Introduction}

Astrocytomas are the most common primary brain tumor types, which, according to the criteria of the World Health Organization (1), are classified to grade I (pilocytic), grade II (diffuse), grade III (anaplastic) and grade IV (glioblastoma multiforme; GBM) $(2,3)$. GBM is the most common central nervous system primary malignancy, which accounts for $60-70 \%$ of all gliomas (4). It is known that GBM may develop de novo or as the consequence of stepwise progression of low-grade or anaplastic astrocytomas $(5,6)$. Multiple cancer-associated factors are known to be involved in the formation and progression of astrocytomas (7-10), of which activated signal transducer and activator of transcription 3 (STAT3) signaling serves pivotal roles in promoting the growth and survival of GBMs by triggering multiple oncogenic signaling cascades (10-12). STAT3 signaling thus emerges as a key initiator and master regulator of malignant transformation of glial cells (13), and the central player in the maintenance and progression of glioblastomas (14-16). Therefore, it would be of clinical values to explore the underlying reason(s) leading to STAT3 activation in stepwise carcinogenesis of GBMs.

It has been recognized that STAT3 signaling transduction can be activated by numerous factors, including extracellular cytokines, growth factors, hormones and oncoproteins $(17,18)$. On the other hand, the data obtained from human and mouse cell lines reveal that the phosphorylation of STAT3 can be negatively regulated in different manners by a group of suppressors, including protein inhibitors of activated stats (PIAS), suppressors of cytokine signaling proteins (SOCS) and $\mathrm{SH} 2$ containing tyrosine phosphatase (SHP1 and SHP2) cascades (19-24). For example, inhibition of PIAS3 results in enhanced proliferation of glioblastoma cells and PIAS3 overexpression inhibits STAT3 transcriptional activity (25). However, no comprehensive in vivo data has been available concerning the expression patterns of those STAT3 negative regulators and their relevance with STAT3 activation in different grades of astrocytomas. The present study aims to address the aforementioned issues. 


\section{Materials and methods}

Glioblastoma specimens and tissue-microarray construction. The archived paraffin tissue blocks of 105 cases of astrocytomas surgical specimens were kindly provided by the doctors at the Department of Clinical Pathology, Anshan Central Hospital (Anshan, China). Prior to experiments, hematoxylin and eosin staining was performed on the sections of those tissue blocks for morphological re-examination. The representative tumor and, where possible, tumor surrounding non-cancerous regions in each of the tissue blocks were determined and marked during the re-examination. These marked samples were used for glioblastoma tissue microarray construction, as previously described (26).

Antibodies and their working concentration. The antibodies used for immunohistochemical staining are as follows: Rabbit anti-human p-STAT3 (Santa Cruz Biotechnology, Inc., Santa Cruz, CA, USA; cat. no. sc-135649; 1:200), rabbit anti-human PIAS3 polyclonal antibody (Bioworld Technology, Inc., St. Louis Park, MN, USA; cat. no. BS1467; 1:200), rabbit anti-human SOCS1 polyclonal antibody (Santa Cruz Biotechnology, Inc.; cat. no. SC9021; 1:180), rabbit anti-human SOCS3 polyclonal antibody (Santa Cruz Biotechnology, Inc.; cat. no. sc-9023; 1:180), rabbit anti-human p-SHP2 polyclonal antibody (Sangon Biotech Co., Ltd., Shanghai, China; cat. no. D155149; 1:150). A DAB Detection kit (streptavidin-biotin; ZSGB-BIO, Beijing, China; cat. no. SP-9000) was used for protein detection.

Immunohistochemical staining. The astrocytomas tissue microarrays in the densities of 56 tissue spots $/ \mathrm{cm}^{2}$ were constructed and subsequently sectioned in series. The 7- $\mu \mathrm{m}$ thick tissue sections were respectively used for p-STAT3, SOCS1, SOCS3, PIAS3 and p-SHP2 oriented immunohistochemical staining, as described previously (27). Briefly, the tissue sections were washed with PBS and incubated in non-immune animal serum working solution blocking buffer for $20 \mathrm{~min}$ at $37^{\circ} \mathrm{C}$. The primary antibody was applied to the tissue sections overnight at $4^{\circ} \mathrm{C}$. Following three washes with PBS, the tissue sections were incubated with a goat anti-rabbit biotin-labeling generic secondary antibody (DAB Detection kit) for $20 \mathrm{~min}$ at $37^{\circ} \mathrm{C}$. Horseradish peroxidase-labeled streptavidin solution was applied (1:500) to the slides for $20 \mathrm{~min}$ at $37^{\circ} \mathrm{C} \cdot 3,3^{\prime}$-Diaminobenzidine (DAB)-staining was detected using a DAB Detection kit, according to the manufacturer's protocol. The array sections without primary antibody incubation were used as background controls. Based on the labeling density, two independent researchers, in a blinded manner, evaluated the staining results and scored them as negative $(-)$, weak $(+)$, moderate $(++)$ or strong positive (+++) (28).

Statistical analysis. Non-parametric Mann-Whitney tests were applied to analyze the expression differences between different grade astrocytoma tissues and non-cancerous brain samples surrounding the tumor. The data were statistically analyzed by Spearman rank and bivariate correlation using SPSS 17.0 software. $\mathrm{P}<0.05$ was considered to indicate a statistically significant difference.

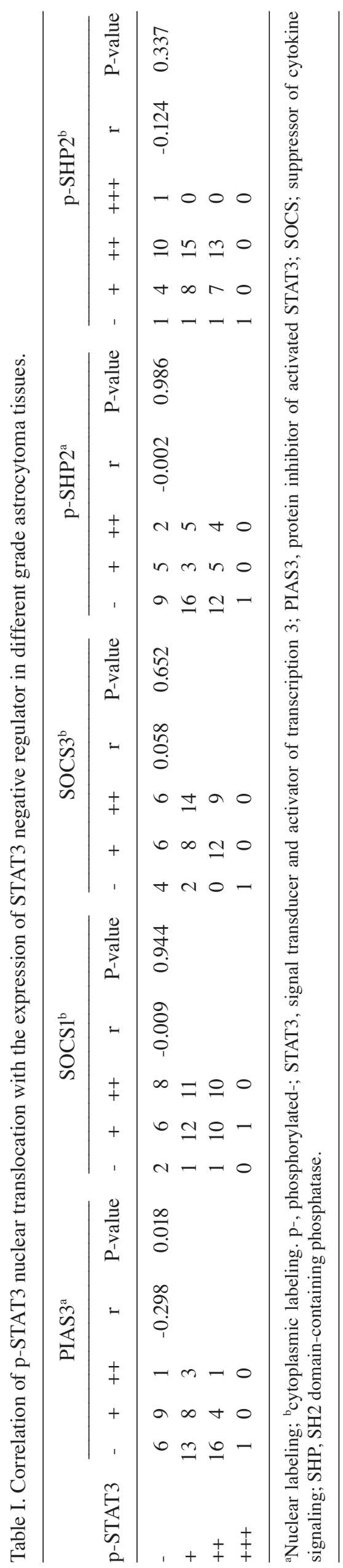




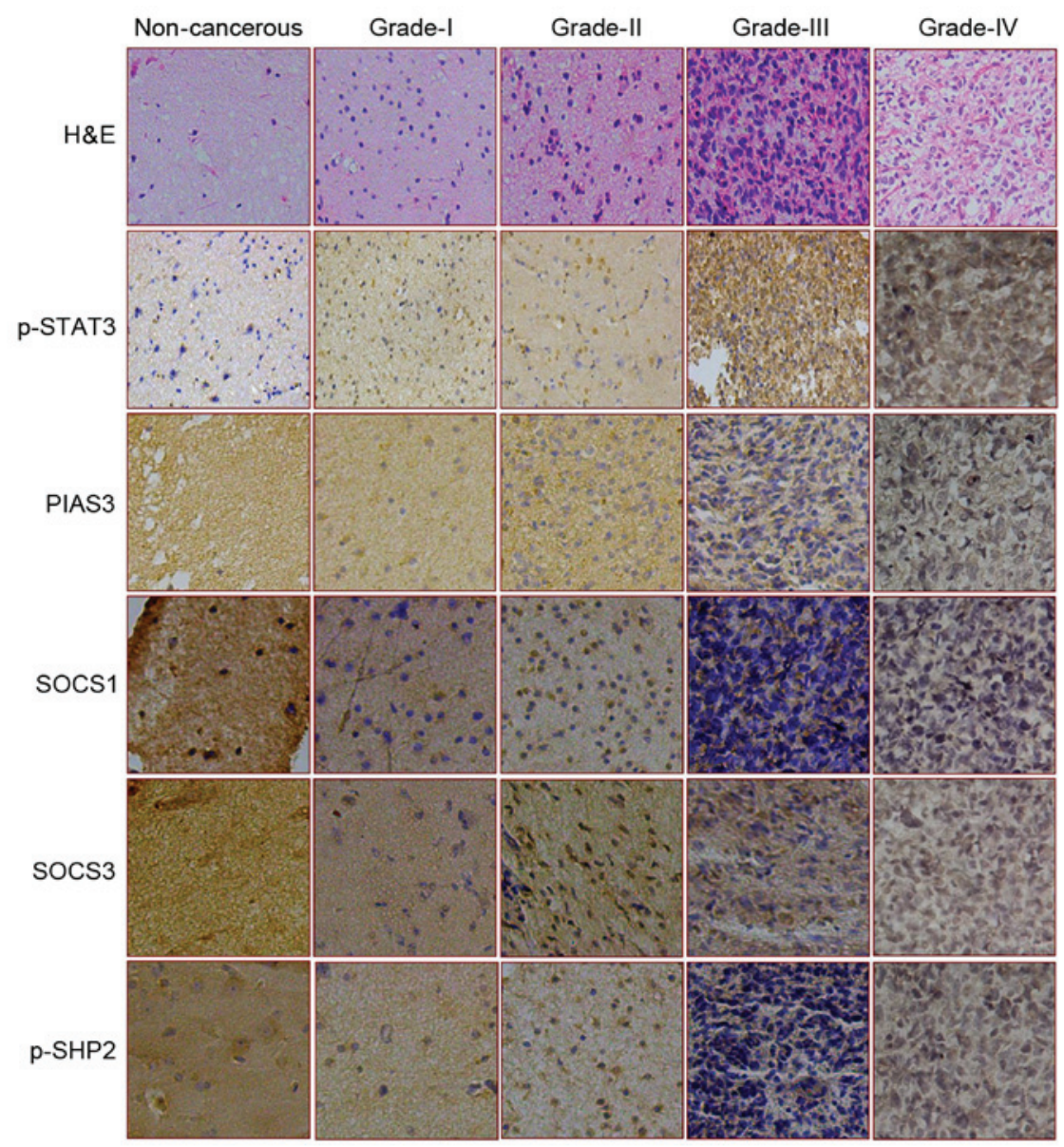

Figure 1. Expression and intracellular distribution patterns of p-STAT3 and its four negative regulators in various brain tissue samples. The expression and distribution of p-STAT3, PIAS3, SOCS1, SOCS4 and p-SHP2 were assessed by immunostaining in non-cancerous brain tissue and four grades of astrocytomas. H\&E staining was also used to look at the cells (magnification, x100). H\&E, hematoxylin and eosin staining; p-, phosphorylated-; STAT3, signal transducer and activator of transcription 3; PIAS3, protein inhibitor of activated STAT3; SOCS, suppressor of cytokine signaling; SHP, SH2 domain-containing phosphatase.

\section{Results and Discussion}

According to the classification criteria of World Health Organization (1), the astrocytoma specimens are classified as grade I (pilocytic), grade II (diffuse), grade III (anaplastic) or grade IV (GBM) $(2,5)$. In the case of GBMs, they may arise primarily (de novo) or are transformed from the lower-grade astrocytomas (29). The primary and secondary GBMs can be classified by several factors including the patient age (5). It has been demonstrated that primary GBMs usually occurs in patients aged $>50$ years, while the secondary glioblastomas are more common among younger patients (30). Of the 94 surgical astrocytomas specimens used in the present study, 23 cases were grouped into grade I (pilocytic), 35 cases to grade II (diffuse), 22 to grade III (anaplastic) and 14 to GBMs. According to the clinical records, 5/14 GBM patients are $>50$ years and, therefore, can be considered as the primary GBMs and the remaining 9 cases as the secondary tumor types. The representative portions of the tumor, as well as tumor surrounding non-cancerous brain tissues of the above specimens, were sampled from the tissue blocks for tissue microarrays construction, as previously described (31). The prepared tissue microarrays in the density of 56 tissue spots/microarray were used for immunohistochemical examination.
A body of evidence demonstrates that STAT3 activation is positively correlated with astrocytomas progression $(25,32,33)$ and is critical for the growth and survival of glioblastoma cells since p-STAT3 proteins trigger or upregulate its downstream gene expression following translocation to the nucleus (34). The results of immunohistochemical staining (Figs. 1 and 2) revealed that p-STAT3 nuclear translocation was rarely observed in non-cancerous brain tissues $(0 / 11 ; 0 \%)$, while the frequencies were increased to $65.2 \%(15 / 23)$ in grade I, $77.1 \%$ (27/35) in grade II, 81.8\% (18/22) in grade III and 85.7\% $(12 / 14)$ in grade IV astrocytomas. p-STAT3 nuclear translocation was observed in all of five GBMs (100\%) from patients $>50$ years and 7/9 GBMs (77.8\%) from patients $>50$ years. Statistical analyses revealed the following: i) The frequencies of p-STAT3 nuclear translocation were significantly increased in the four subtypes of astrocytomas compared with that of the non-cancerous brain tissues and ii) that the incidences of p-STAT3 nuclear translocation are closely correlated with tumor grading [Spearman rank and bivariate correlation $\left.\left(r_{s}\right)=0.207, P=0.045\right]$. These results further demonstrated the potential promoting effects of STAT3 signaling in the stepwise progress of astrocytomas and de novo formation of GBMs. Further investigation of the underlying reasons leading to the disordered STAT3 activation is of potential prognostic and therapeutic value in the management of astrocytomas. 

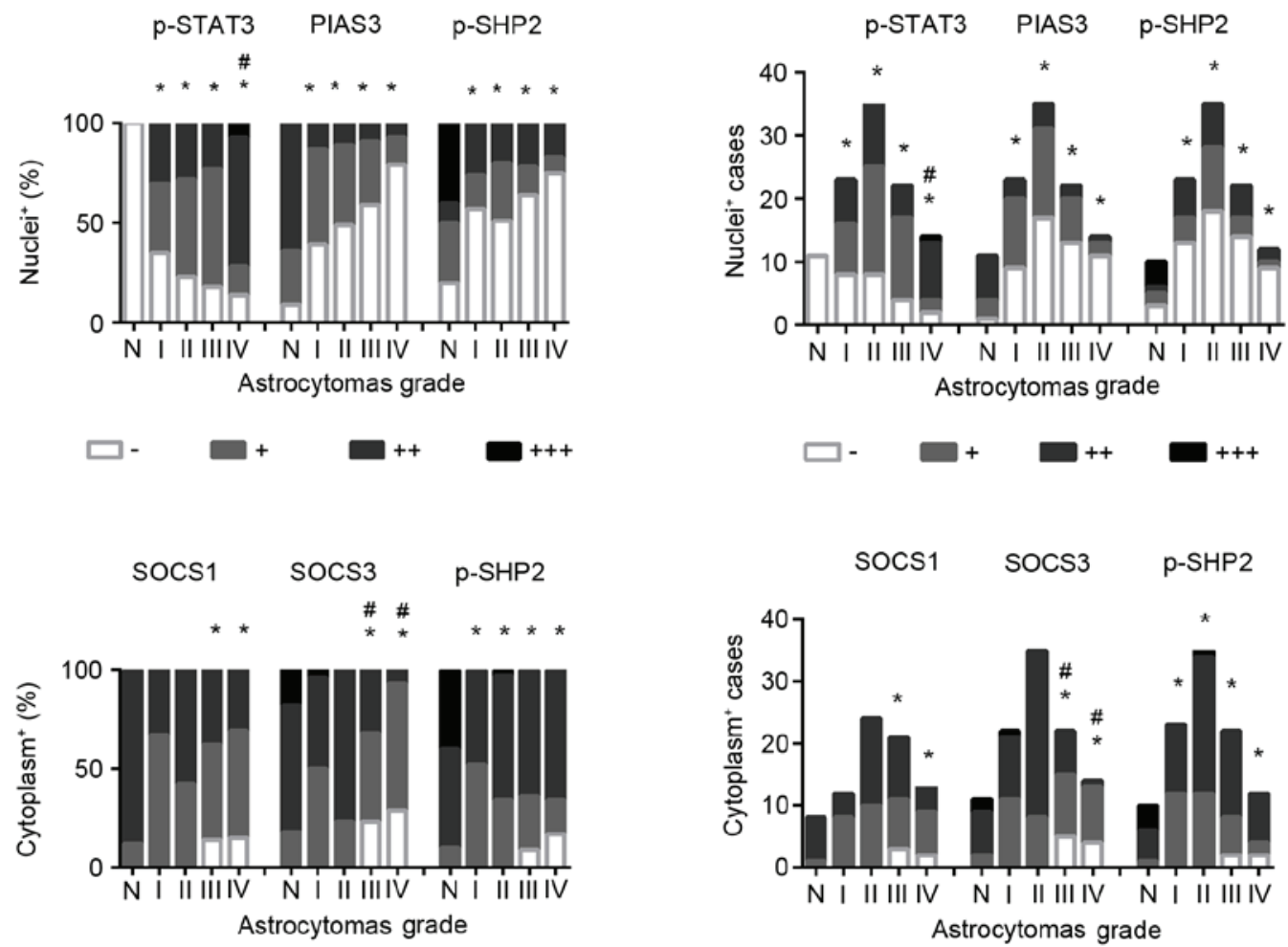

Figure 2. Fractionation of p-STAT3 nuclear translocation and the expression levels of the four STAT3 negative regulators in various brain tissue samples. The presence of p-STAT3, PIAS3 and p-SHP2 in the nuclei and cytoplasm was assessed in the $\mathrm{N}$ tissue and four grades (I, II, III and IV) of astrocytomas ("P<0.05 compared with $\mathrm{N}$; "P<0.05 compared with different grades of astrocytomas tissues). p-, phosphorylated-; STAT3, signal transducer and activator of transcription 3; PIAS3, protein inhibitor of activated STAT3; SOCS, suppressor of cytokine signaling; SHP, SH2 domain-containing phosphatase.

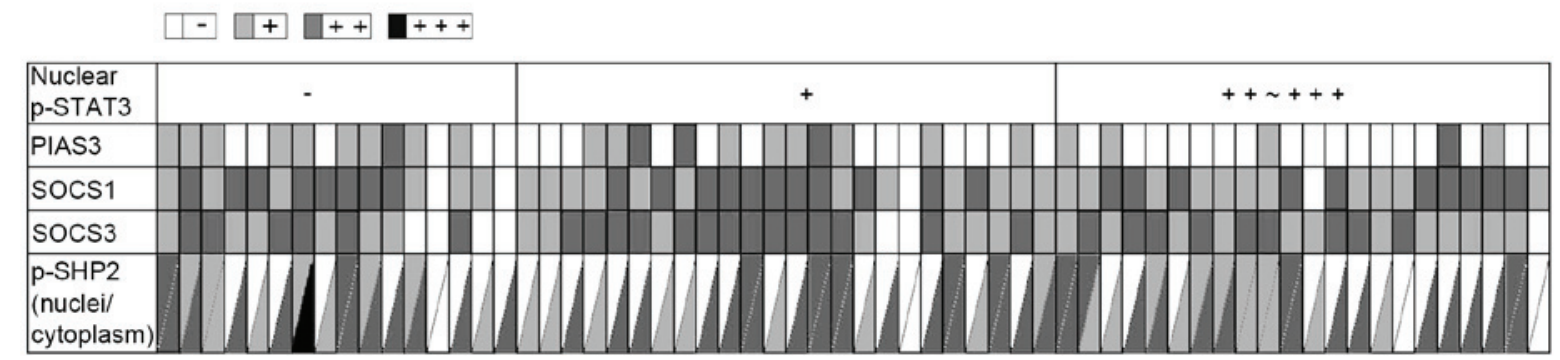

Figure 3. Relevance of p-STAT3 nuclear localization with the expression levels of PIAS3, SOCS1, SOCS3 and p-SHP2 in astrocytoma tissues. The staining results were scored as negative (-), weak (+), moderate (++) or strong positive (+++), and are indicated as white, light gray, medium grey and black, respectively. Each small grid represents an array point and each vertical represents different antibody expression in the same array point. p-, phosphorylated-; STAT3, signal transducer and activator of transcription 3; PIAS3, protein inhibitor of activated STAT3; SOCS, suppressor of cytokine signaling; SHP, SH2 domain-containing phosphatase.

It has been recognized that PIAS3 functions as a negative regulator of STAT-3 signaling by interfering with the interaction between p-STAT3 and its target genes (19). In agreement with the above notion, the present in vitro data revealed upregulated expression and increased nuclear translocation of PIAS3 in resveratrol-suppressed glioblastoma cells, accompanied by STAT3 inactivation (35). However, no data concerning the status of PIAS3 in different grades of astrocytomas has been thus far available, although the activated STAT3 signaling has been frequently observed in astrocytomas (15). As shown in Fig. 2, higher levels (++ and +++) of PIAS3 expression were observed in $63.6 \%$ (7/11) of tumor surrounding brain tissues, which is reduced to $13.1 \%$ in grade I (3/23), $11.4 \%$ in grade II $(4 / 5), 9.1 \%$ in grade III (2/22) and $7.1 \%$ in grade IV (1/14) astrocytomas. Accordingly, distinct PIAS3 nuclear labeling is observed in the non-cancerous, but not in the majority (54\%) of tumor tissues (Figs. 1-3). Statistical analyses revealed significant differences of PIAS3 detection rates between the four subtypes of astrocytomas and the non-cancerous brain tissues, and the negative correlation of PIAS3 expression with astrocytomas formation $(\mathrm{P}<0.05)$. Furthermore, the expression of PIAS3 was negatively-correlated with STAT3 nuclear translocation $\left(r_{s}=-0.298 ; P=0.018\right.$; Table I). These results together with our aforementioned in vitro findings indicated that PIAS3 may serve negative roles in regulating STAT3 signaling in glioblastoma cells in vitro and in vivo. Alternatively, PIAS3 downregulation in the four subtypes of astrocytomas may results in STAT3 activation or indirectly 
enhance the biological effects of the upstream STAT3 activators (15). In this context, the decreased expression of PIAS3 and the lack of PIAS3 nuclear translocation is an unfavorable prognostic factor of astrocytomas.

SOCS1 and SOCS3 are the predominant members of the SOCS protein family, which work in a classic negative feedback loop to attenuate STAT3 activity by suppressive binding with phosphorylated JAK and/or facilitating ubiqitination of JAK in the cytoplasm (21). SOCS1 and SOCS3 expression in GBMs can be epigenetically regulated in the form of hypermethylation in $\mathrm{CpG}$ island $(36,37)$. For instance, the methylation of SOCS3 appears to be involved in the pathogenesis of GBMs and in the resistance of GBMs to conventional anticancer drugs (38). However, the correlation of SOCS1 and SOCS3 downregulation with STAT3 activation in human astrocytomas remains to be reported. The present IHC results revealed that SOCS1 and SOCS3 are expressed in higher levels (++ and +++$)$ in the non-cancerous specimens assessed, while their levels are decreased (+ or -) in the astrocytomas tissues (Figs. 1-3). Statistical analyses demonstrated that SOCS3 $\left(\mathrm{r}_{\mathrm{s}}=-0.400 ; \mathrm{P}=0.000\right)$, rather than SOCS1 downregulation $\left(r_{s}=-0.160 ; \mathrm{P}=0.187\right)$, is negatively correlated with the tumor grading (Fig. 2). Nevertheless, neither SOCS1 nor SOCS3 expression pattern is statistically correlated with p-STAT3 nuclear translocation $\left(r_{\mathrm{s}}=-0.009, \mathrm{P}=0.944 ; \mathrm{r}_{\mathrm{s}}=-0.058\right.$, $\mathrm{P}=0.652$ ). It has been reported that SOCS3 inactivation by promoter hypermethylation is mutually exclusive to EGFR activation in glioblastomas and preferentially promotes glioma cell invasion through the activation of STAT3 and FAK (39). Therefore, it would be possible that the epigenetically downregulated SOCS3 and SOCS1 may confer on GBM cells more aggressive biological behaviors, although the relevance of their downregulation with STAT3 activation cannot be totally ruled out at present stage.

SHP2 is a non-receptor type protein tyrosine phosphatase (40) and its phosphorylated form (p-SHP2) downregulates STAT3 activation by dephosphorylating active STAT3 complexes both in the cytoplasm and in the nucleus (41). The statuses of SHP2 and their relevance with STAT3 signaling in GBMs have been reported with differeing opinions $(42,43)$. It was revealed that SHP2-mediated antagonism of STAT3 phosphorylation prevails in the promotion of GBM cell death in response to EGFR and c-MET co-inhibition (42), while SHP2 can promote glioblastoma cell growth by suppression of cellular senescence (43). The present immunohistochemical results using a p-SHP2 specific antibody revealed that cytoplasmic p-SHP2 staining (++ and +++$)$ was observed in all of the non-cancerous specimens examined, of which 8 cases $(8 / 10 ; 80 \%)$ were found with p-SHP2 nuclear translocation (Figs. 1-3). In the case of astrocytomas, the detection rates of cytoplasmic p-SHP2 are not changed distinctly, but the frequencies of nuclear p-SHP2 detection are remarkably decreased in the tumor tissues, in particular in grade III (36.4\%, 8/22) and grade IV (25\%, 3/12) (Figs. 1 and 3). However, the statistical analyses revealed no correlation of nuclear translocation $\left(\mathrm{r}_{\mathrm{s}}=-0.106, \mathrm{P}=0.315\right)$ and cytoplasmic staining $\left(\mathrm{r}_{\mathrm{s}}=0.065, \mathrm{P}=0.536\right)$ of $\mathrm{p}-\mathrm{SHP} 2$ with astrocytomas grading and $\mathrm{p}-\mathrm{STAT} 3$ nuclear translocation (nuclei, $\mathrm{r}_{\mathrm{s}}=-0.002$ and $\mathrm{P}=0.986$; cytoplasm, $\mathrm{r}_{\mathrm{s}}=-0.124$ and $\mathrm{P}=0.337$; Fig. 2; Table I). Although the present findings may implicate that the reductive tendencies of p-SHP2 level and nuclear translocation may be favorable for astrocytomas formation presumably via preventing cell death (43) and/or reinforcing STAT3 activation caused by STAT3 activator overexpression and PIAS3 reduction (25).

In conclusion, SOCS1, SOCS3, PIAS3 and p-SHP2 expression patterns and the frequencies of phosphorylated STAT3/p-STAT3 nuclear translocation in non-cancerous brain tissues and the four grades of astrocytomas were profiled by tissue microarray-based immunohistochemical staining. The results revealed that p-STAT3 nuclear translocation is progressively common as the tumor grades increase. By contrast, the expression levels of SOCS1, SOCS3, PIAS3 and p-SHP2 tended to decrease as the tumor progressed. Statistical analyses revealed that downregulation of PIAS3 is more correlated with p-STAT3 nuclear translocation compared with other STAT3 negative regulators. As a result of the importance of STAT3 activation for the growth and survival of glioblastoma cells, the decreased expression of PIAS3 can be regarded as an unfavorable prognostic factor of astrocytomas patients. SOCS1, SOCS3 and p-SHP2 downregulation, and p-SHP2 nuclear translocation in astrocytomas tissues must have certain biological implications and it would be of value to further investigate.

\section{Acknowledgements}

The authors would like to thank the doctors in the Department of Clinical Pathology of An-Shan First Hospital for their co-operation in sample collection and pathological consultation. This study was supported by grants from the National Natural Science Foundation of China (nos. 81450016, 81272786 and 30971038), the research fund for PhD supervisors from National Education Department of China (no. 20122105110005), the Program for Changjiang Scholar and Innovative Research Team in University (PCSIRT; IRT13049), the Liaoning Department of Education for key laboratory (no. L2012317 and L20133453) and the Natural Science Foundation of Liaoning Province (no. 2013023040 and 2013023050).

\section{References}

1. Kros JM: Grading of gliomas: The road from eminence to evidence. J Neuropathol Exp Neurol 70: 101-109, 2011.

2. Louis DN, Holland EC and Cairncross JG: Glioma classification: A molecular reappraisal. Am J Pathol 159: 779-786, 2001

3. Suryadevara CM, Verla T, Sanchez-Perez L, Reap EA, Choi BD, Fecci PE and Sampson JH: Immunotherapy for malignant glioma. Surg Neurol Int 6 (Suppl 1): S68-S77, 2015.

4. Wen PY and Kesari S: Malignant gliomas in adults. N Engl J Med 359: 492-507, 2008.

5. Jovcevska I, Kocevar N and Komel R: Glioma and glioblastoma-how much do we (not) know? Mol Clin Oncol 1: 935-941, 2013.

6. OhgakiH: Genetic pathways to glioblastomas. Neuropathology 25 : $1-7,2005$.

7. Zhong Z, Wen Z and Darnell JE Jr: Stat3: A STAT family member activated by tyrosine phosphorylation in response to epidermal growth factor and interleukin-6. Science 264: 95-98, 1994.

8. Rahaman SO, Vogelbaum MA and Haque SJ: Aberrant Stat3 signaling by interleukin-4 in malignant glioma cells: Involvement of IL-13Ralpha2. Cancer Res 65: 2956-2963, 2005.

9. Liu Q, Li G, Li R, Shen J, He Q, Deng L, Zhang C and Zhang J: IL-6 promotion of glioblastoma cell invasion and angiogenesis in U251 and T98G cell lines. J Neurooncol 100: 165-176, 2010. 
10. Jackson C, Ruzevick J, Amin AG and Lim M: Potential role for STAT3 inhibitors in glioblastoma. Neurosurg Clin N Am 23: 379-389, 2012

11. Johnston PA and Grandis JR: STAT3 signaling: Anticancer strategies and challenges. Mol Interv 11: 18-26, 2011.

12. See AP, Han JE, Phallen J, Binder Z, Gallia G, Pan F, Jinasena D, Jackson C, Belcaid Z, Jeong SJ, et al: The role of STAT3 activation in modulating the immune microenvironment of GBM. J Neurooncol 110: 359-368, 2012.

13. Carro MS, Lim WK, Alvarez MJ, Bollo RJ, Zhao X, Snyder EY, Sulman EP, Anne SL, Doetsch F, Colman H, et al: The transcriptional network for mesenchymal transformation of brain tumours. Nature 463: 318-325, 2010.

14. Luwor RB, Stylli SS and Kaye AH: The role of Stat 3 in glioblastoma multiforme. J Clin Neurosci 20: 907-911, 2013.

15. Mizoguchi M, Betensky RA, Batchelor TT, Bernay DC Louis DN and Nutt CL: Activation of STAT3, MAPK and AKT in malignant astrocytic gliomas: Correlation with EGFR status, tumor grade, and survival. J Neuropathol Exp Neurol 65 : $1181-1188,2006$.

16. Abou-Ghazal M, Yang DS, Qiao W, Reina-Ortiz C, Wei J, Kong LY, Fuller GN, Hiraoka N, Priebe W, Sawaya R and Heimberger AB: The incidence, correlation with tumor-infiltrating inflammation and prognosis of phosphorylated STAT3 expression in human gliomas. Clin Cancer Res 14: 8228-8235, 2008.

17. Levy DE and Darnell JE Jr: Stats: Transcriptional control and biological impact. Nat Rev Mol Cell Biol 3: 651-662, 2002.

18. Dinasarapu AR, Gupta S, Ram Maurya M, Fahy E, Min J, Sud M, Gersten MJ, Glass CK and Subramaniam S: A combined omics study on activated macrophages-enhanced role of STATs in apoptosis, immunity and lipid metabolism. Bioinformatics 29 : 2735-2743, 2013.

19. Chung CD, Liao J, Liu B, Rao X, Jay P, Berta P and Shuai K: Specific inhibition of Stat3 signal transduction by PIAS3. Science 278: 1803-1805, 1997.

20. Shuai K: Regulation of cytokine signaling pathways by PIAS proteins. Cell Res 16: 196-202, 2006.

21. Zhou H, Miki R, Eeva M, Fike FM, Seligson D, Yang L, Yoshimura A, Teitell MA, Jamieson CA and Cacalano NA: Reciprocal regulation of SOCS 1 and SOCS3 enhances resistance to ionizing radiation in glioblastoma multiforme. Clin Cancer Res 13: 2344-2353, 2007.

22. Kim DJ, Tremblay ML and Digiovanni J: Protein tyrosine phosphatases, TC-PTP, SHP1 and SHP2, cooperate in rapid dephosphorylation of Stat 3 in keratinocytes following UVB irradiation. PloS One 5: e10290, 2010.

23. Funato K, Yamazumi Y, Oda T and Akiyama T: Tyrosine phosphatase PTPRD suppresses colon cancer cell migration in coordination with CD44. Exp Ther Med 2: 457-463, 2011.

24. Bixler SL, Sandler NG, Douek DC and Mattapallil JJ: Suppressed Th17 levels correlate with elevated PIAS3, SHP2, and SOCS3 expression in CD4 T cells during acute simian immunodeficiency virus infection. J Virol 87: 7093-7101, 2013.

25. Brantley EC, Nabors LB, Gillespie GY, Choi YH, Palmer CA, Harrison K, Roarty K and Benveniste EN: Loss of protein inhibitors of activated STAT-3 expression in glioblastoma multiforme tumors: Implications for STAT-3 activation and gene expression. Clin Cancer Res 14: 4694-4704, 2008.

26. Li H, Sun Y, Kong QY, Zhang KL, Wang XW, Chen XY, Wang Q and Liu J: Combination of nucleic acid and protein isolation with tissue array construction: Using defined histologic regions in single frozen tissue blocks for multiple research purposes. Int $J$ Mol Med 12: 299-304, 2003.
27. Li H, Guo L, Li JW, Liu N, Qi R and Liu J: Expression of hyaluronan receptors CD44 and RHAMM in stomach cancers: Relevance with tumor progression. Int J Oncol 17: 927-932, 2000.

28. Ma JX, Li H, Chen XM, Yang XH, Wang Q, Wu ML, Kong QY, Li ZX and Liu J: Expression patterns and potential roles of SIRT1 in human medulloblastoma cells in vivo and in vitro. Neuropathology 33: 7-16, 2013.

29. Bralten LB and French PJ: Genetic alterations in glioma. Cancers 3): 1129-1140, 2011.

30. Yoshida J: Molecular neurosurgery using gene therapy to treat malignant glioma. Nagoya J Med Sci 59: 97-105, 1996.

31. Xia SL, Wu ML, Li H, Wang JH, Chen NN, Chen XY, Kong QY, Sun Z and Liu J: CRABP-II- and FABP5-independent responsiveness of human glioblastoma cells to all-trans retinoic acid. Oncotarget 6: 5889-5902, 2015.

32. Lo HW, Cao X, Zhu H and Ali-Osman F: Constitutively activated STAT3 frequently coexpresses with epidermal growth factor receptor in high-grade gliomas and targeting STAT3 sensitizes them to Iressa and alkylators. Clin Cancer Res 14: 6042-6054, 2008.

33. Schaefer LK, Ren Z, Fuller GN and Schaefer TS: Constitutive activation of Stat3alpha in brain tumors: Localization to tumor endothelial cells and activation by the endothelial tyrosine kinase receptor (VEGFR-2). Oncogene 21: 2058-2065, 2002.

34. O'Shea JJ, Schwartz DM, Villarino AV, Gadina M, McInnes IB and Laurence A: The JAK-STAT pathway: Impact on human disease and therapeutic intervention. Annu Rev Med 66: 311-328, 2015.

35. Shu XH, Li H, Sun XX, Wang Q, Sun Z, Wu ML, Chen XY, Li C, Kong QY and Liu J: Metabolic patterns and biotransformation activities of resveratrol in human glioblastoma cells: Relevance with therapeutic efficacies. PloS One 6: e27484, 2011.

36. Tamiya T, Kashiwagi I, Takahashi R, Yasukawa $H$ and Yoshimura A: Suppressors of cytokine signaling (SOCS) proteins and JAK/STAT pathways: Regulation of T-cell inflammation by SOCS1 and SOCS3. Arterioscler Thromb Vasc Biol 31: 980-985, 2011.

37. Sutherland KD, Lindeman GJ, Choong DY, Wittlin S, Brentzell L, Phillips W, Campbell IG and Visvader JE: Differential hypermethylation of SOCS genes in ovarian and breast carcinomas. Oncogene 23: 7726-7733, 2004.

38. Martini M, Pallini R, Luongo G, Cenci T, Lucantoni C and Larocca LM: Prognostic relevance of SOCS3 hypermethylation in patients with glioblastoma multiforme. Int J Cancer 123: 2955-2960, 2008.

39. Lindemann C, Hackmann O, Delic S, Schmidt N, Reifenberger G and Riemenschneider MJ: SOCS3 promoter methylation is mutually exclusive to EGFR amplification in gliomas and promotes glioma cell invasion through STAT3 and FAK activation. Acta Neuropathol 122: 241-251, 2011.

40. Feng GS: Shp2-mediated molecular signaling in control of embryonic stem cell self-renewal and differentiation. Cell Res 17: 37-41, 2007.

41. Rakesh K and Agrawal DK: Controlling cytokine signaling by constitutive inhibitors. Biochem Pharmacol 70: 649-657, 2005.

42. Furcht CM, Buonato JM, Skuli N, Mathew LK, Muñoz Rojas AR, Simon MC and Lazzara MJ: Multivariate signaling regulation by SHP2 differentially controls proliferation and therapeutic response in glioma cells. J Cell Sci 127: 3555-3567, 2014.

43. Sturla LM, Zinn PO, Ng K, Nitta M, Kozono D, Chen CC and Kasper EM: Src homology domain-containing phosphatase 2 suppresses cellular senescence in glioblastoma. Br J Cancer 105: $1235-1243,2011$ 